\title{
Chaos control strategy for a fractional-order financial model
}

\author{
Changjin $\mathrm{Xu}^{1 *} \mathbb{D}$, Chaouki Aouiti ${ }^{2}$, Maoxin Liao ${ }^{3 *}$, Peiluan $\mathrm{Li}^{4}$ and Zixin Liu ${ }^{5}$
}

"Correspondence: xcj403@126.com; maoxinliao@163.com

${ }^{1}$ Guizhou Key Laboratory of Economics System Simulation, Guizhou University of Finance and Economics, Guiyang 550004,

P.R. China

${ }^{3}$ School of Mathematics and Physics, University of South China, Hengyang 421001, P.R. China Full list of author information is available at the end of the article

\section{Springer}

\begin{abstract}
In this paper, we propose a new fractional-order financial model which is a generalized version of the financial model reported in the previous publications. By applying a suitable time-delayed feedback controller, we have control for the chaotic behavior of the fractional-order financial model. We investigate the stability and the existence of a Hopf bifurcation of the fractional-order financial model. A new sufficient condition that guarantees the stability and the existence of a Hopf bifurcation for a fractional-order delayed financial model is presented by regarding the delay as bifurcation parameter. The investigation shows that the delay and the fractional order have an important effect on the stability and Hopf bifurcation of involved model. Some simulations justifying the validity of the derived analytical results are given. The obtained results of this article are innovative and are of great significance in handling the financial issues.
\end{abstract}

MSC: 34C23; 34K18; 37K15; 39A11; 92B20

Keywords: Chaos control; Financial model; Stability; Hopf bifurcation; Fractional order; Delay

\section{Introduction}

In modern society, the study on complex dynamics of financial systems has become a topic of focus in the microeconomic and macroeconomic fields. Numerous researchers pay much attention to this theme. For example, Gao and $\mathrm{Ma}$ [1] considered the chaos and Hopf bifurcation of a finance model. Ma and Chen [2, 3] investigated the bifurcation phenomenon and the global character for a nonlinear finance model. Ma and Wang discussed the Hopf bifurcation and topological horseshoe for a chaotic finance model.

Chaotic phenomenon often occur in many economics. Serletic [4] point out that chaos stands for a radical change of perspective on business cycles. In many cases, chaos will cause the instability of economical systems. Thus chaos control has become an important problem that must be solved for real human life. Generally speaking, the aim of chaotic control is to stabilize a chaotic attractor to an equilibrium point or a periodic solution. In recent years, there have been two main ways to control chaos. One was developed by Ott et al. [5] and another is the time-delayed feedback method proposed by Pyragas [6]. The latter is more convenient than the former. So we adopt the latter to control chaos in this article.

(c) The Author(s) 2020. This article is licensed under a Creative Commons Attribution 4.0 International License, which permits use sharing, adaptation, distribution and reproduction in any medium or format, as long as you give appropriate credit to the original author(s) and the source, provide a link to the Creative Commons licence, and indicate if changes were made. The images or other third party material in this article are included in the article's Creative Commons licence, unless indicated otherwise in a credit line to the material. If material is not included in the article's Creative Commons licence and your intended use is not permitted by statutory regulation or exceeds the permitted use, you will need to obtain permission directly from the copyright holder. To view a copy of this licence, visit http://creativecommons.org/licenses/by/4.0/ 
Fractional calculus has a history of over 300 years. The investigation progress on fractional calculus is very slow due to the lack of theoretical basis and realistic background. In recent years, fractional differential equations have been proved to be potentially useful in electroanalytical chemistry, robotics, bioengineering, viscoelasticity, medicine and so on [7-48]. Different from integer-order models, fractional-order models possess memory, namely, the fractional-order model depends on the history of the model.

In 2016, Yang et al. [49] studied the following financial model:

$$
\left\{\begin{array}{l}
\frac{d u_{1}}{d t}=a_{1}\left(u_{1}+u_{2}\right), \\
\frac{d u_{2}}{d t}=-u_{2}-a_{1} u_{1} u_{3} \\
\frac{d u_{3}}{d t}=a_{2}+a_{1} u_{1} u_{2},
\end{array}\right.
$$

where $a_{i}>0(i=1,2)$ is the constant, $u_{1}$ represents the interest rate, $u_{2}$ represents investment demand and $u_{3}$ represents the price index. Based on the model (1.1) and considering that fractional differential equations have memory and hereditary properties, for practical dynamical process, we can modify system (1.1) as a fractional-order version:

$$
\left\{\begin{array}{l}
\frac{d^{p} u_{1}}{d t^{p}}=a_{1}\left(u_{1}+u_{2}\right), \\
\frac{d^{p} u_{2}}{d t^{p}}=-u_{2}-a_{1} u_{1} u_{3}, \\
\frac{d^{p} u_{3}}{d t^{p}}=a_{2}+a_{1} u_{1} u_{2},
\end{array}\right.
$$

where $p$ represents the fractional order. In economic operation, the interest rate, investment demand and price index are under the impact of their memories. In addition, the process of economic operation has a close connection with the whole time information of the financial system. The model (1.2) has memory and hereditary properties for practical dynamical process, so we think that the model (1.2) shows some novelty and it is better than model (1.1). When $p=0.8$ and $a_{1}=1.79, a_{2}=4$, system (1.2) is chaotic, which is shown in Fig. 1.

The main object of this paper is to discuss two topics: (1) designing a suitable timedelayed feedback controller to suppress the chaos of the system (1.2) and (2) the effect of time delay and the fractional order on the stability and the existence of Hopf bifurcation of controlled system are presented. During the past decades, the time-delayed feedback control technique has only been applied to the control of chaos and Hopf bifurcation of integer-order differential dynamical systems. There are relatively few works that deal with chaos and Hopf bifurcation control by applying time-delayed feedback controllers. Considering the introduction of fractional order for delayed differential systems, the corresponding characteristic equation will be more complex. Thus it is more difficult to analyze the distribution of roots of the characteristic equation of the involved fractional-order dynamical systems. The contributions of this article lie in four aspects:

- The integer-order delayed financial model has been extended to a delayed fractional-order financial model, which can better describe the memory properties of the model.

- The control technique is more complex than that for integer-order differential systems due to the introduction of the fractional order. A set of sufficient conditions that 

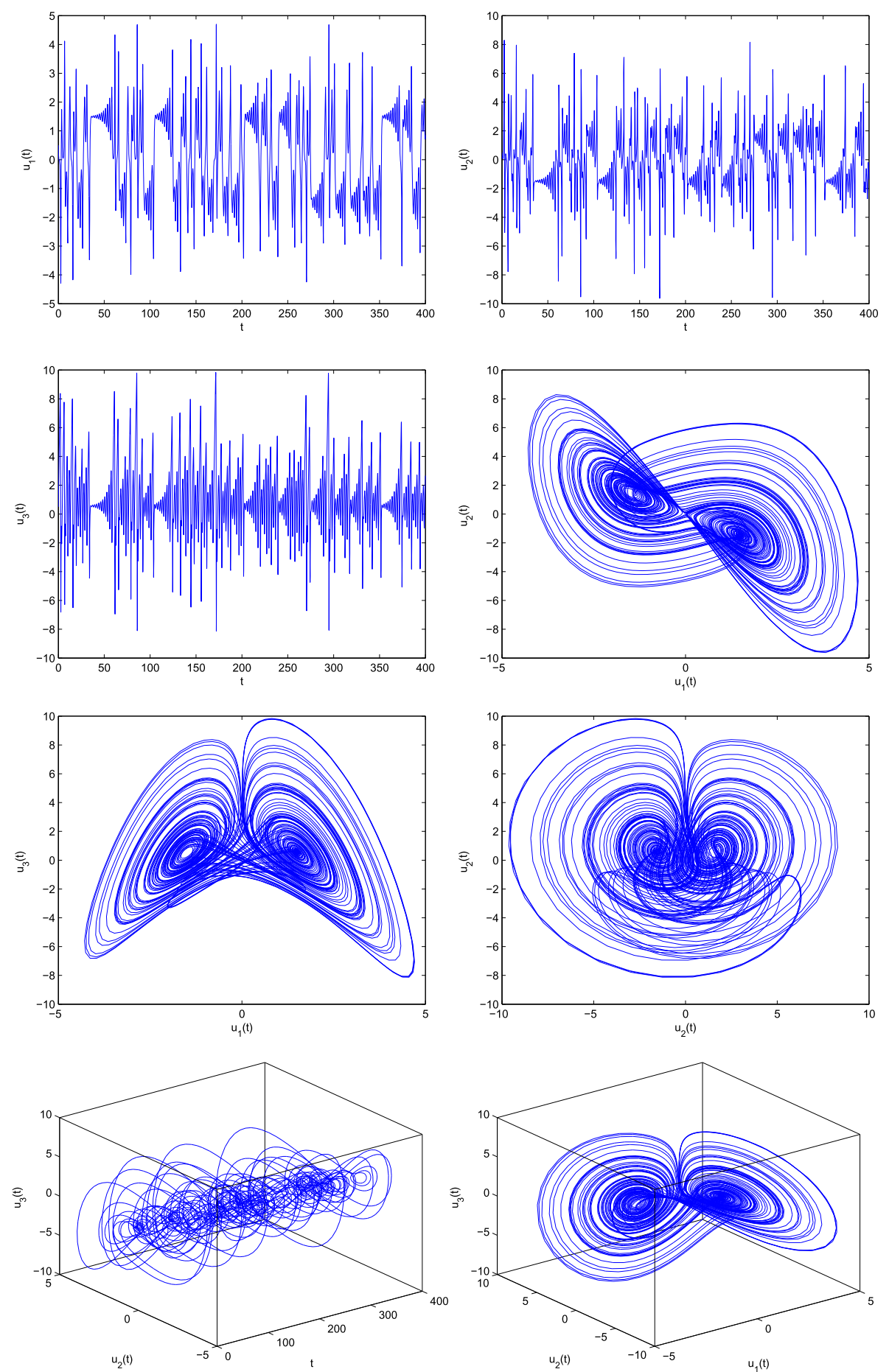

Figure 1 Time history plots, variable relation plots and phase diagrams of system (1.2) with $a_{1}=1.79, a_{2}=4$

ensure the stability and the existence of Hopf bifurcation of the fractional-order delayed financial model are established. The study shows that the delay and fractional order have an important effect on the stability and the existence of Hopf bifurcation of involved controlled systems. 
- Up to now, there are few papers that focus on the Hopf bifurcation of fractional-order delayed financial model. The theoretical findings of this article will enrich and develop the Hopf bifurcation theory of fractional-order delayed differential equations and supplement the earlier publications.

- The approach of this paper can provide a good reference in the study of some similar fractional-order delayed differential models.

The rest of this paper is organized as follows. In Sect. 2, several definitions and lemmas on fractional calculus are given. In Sect. 3, a time-delay feedback controller is designed to control the chaos of the chaotic fractional-order financial model. In Sect. 4, a numerical example is given to check the theoretical predictions. Finally, a brief conclusion is included.

\section{Preliminary results}

In this section, two definitions and two lemmas of fractional calculus are introduced.

Definition 2.1 ([50]) The fractional integral of order $\delta$ for a function $h(\xi)$ is defined as follows:

$$
\mathcal{I}^{\delta} h(\xi)=\frac{1}{\Gamma(\delta)} \int_{\xi_{0}}^{\xi}(\xi-s)^{\delta-1} h(s) d s
$$

where $\xi \geq \xi_{0}, \delta>0, \Gamma(\cdot)$ denotes the Gamma function and $\Gamma(s)=\int_{0}^{\infty} \xi^{s-1} e^{-\xi} d \xi$.

Definition 2.2 ([50]) The Caputo fractional-order derivative of order $\delta$ for a function $h(\xi) \in\left(\left[\xi_{0}, \infty\right), R\right)$ is defined as follows:

$$
\mathcal{D}^{\delta} h(\xi)=\frac{1}{\Gamma(n-\delta)} \int_{\xi_{0}}^{\xi} \frac{h^{(n)}(s)}{(\xi-s)^{\delta-n+1}} d s,
$$

where $\xi \geq \xi_{0}$ and $n$ is a positive integer such that $n-1 \leq \delta<n$. In particular, when $0<\delta<1$,

$$
\mathcal{D}^{\delta} h(\xi)=\frac{1}{\Gamma(1-\delta)} \int_{\xi_{0}}^{\xi} \frac{h^{\prime}(s)}{(\xi-s)^{\delta}} d s
$$

Lemma 2.1 ([51]) Let there be given an autonomous system $\mathcal{D}^{\delta} z=\mathcal{A} z, z(0)=z_{0}$ where $0<\delta<1, z \in R^{n}, \mathcal{A} \in R^{n \times n}$. Suppose that $\lambda_{i}(i=1,2, \ldots, n)$ is the root of the characteristic equation of $\mathcal{D}^{\delta} z=\mathcal{A} z$. Then system $\mathcal{D}^{\delta} z=\mathcal{A} z$ is asymptotically stable $\Leftrightarrow\left|\arg \left(\lambda_{i}\right)\right|>\frac{\delta \pi}{2}$ $(i=1,2, \ldots, n)$. Especially, this system is stable $\Leftrightarrow\left|\arg \left(\lambda_{i}\right)\right|>\frac{\delta \pi}{2}(i=1,2, \ldots, n)$ and those critical eigenvalues that satisfy $\left|\arg \left(\lambda_{i}\right)\right|=\frac{\delta \pi}{2}(i=1,2, \ldots, n)$ possess geometric multiplicity one.

Lemma 2.2 ([8]) For the given fractional-order delayed differential equation with Caputo derivative: $\mathcal{D}^{\delta} u(t)=\mathcal{C}_{1} u(t)+\mathcal{C}_{2} u(t-\varrho)$, where $u(t)=\phi(t), t \in[-\varrho, 0], \delta \in(0,1], u \in R^{n}$, $\mathcal{C}_{1}, \mathcal{C}_{2} \in R^{n \times n}, \varrho \in R^{+(n \times n)}$. Then the characteristic equation of the system is $\operatorname{det} \mid s^{\delta} I-\mathcal{C}_{1}-$ $\mathcal{C}_{2} e^{-s e} \mid=0$. If all the roots of the characteristic equation of the system have negative real roots, then the zero solution of the system is asymptotically stable. 


\section{Controller design for chaos control}

Over the past few decades, many linear time-delay feedback methods are applied to control the Hopf bifurcation of integer-order models. However, the linear time-delay feedback controllers are very rare in controlling a Hopf bifurcation of fractional-order models. To make up for the deficiency, we design a linear time-delay feedback controller [52] which takes the form

$$
\kappa_{i}\left[u_{i}(t)-u_{i}(t-\varrho)\right] \quad(i=1,2)
$$

where $\kappa_{i}(i=1,2)$ is the feedback strength and $\varrho$ is the time delay. $\kappa_{i}, \varrho \in R$ and $\varrho \geq 0$. Clearly, system (1.2) has two equilibrium points,

$$
E_{1}\left(-\sqrt{\frac{a_{2}}{a_{1}}}, \sqrt{\frac{a_{2}}{a_{1}}}, \frac{1}{a_{1}}\right), \quad E_{2}\left(\sqrt{\frac{a_{2}}{a_{1}}},-\sqrt{\frac{a_{2}}{a_{1}}}, \frac{1}{a_{1}}\right) .
$$

In this paper, we only consider the equilibrium point $E_{1}$ and $E_{2}$ can be handled in a similar approach. Adding the time-delayed feedback controller $\kappa_{i}\left[u_{i}(t)-u_{i}(t-\varrho)\right]$ to the $i$ th equation of system (1.2), we have

$$
\left\{\begin{array}{l}
\frac{d u_{1}^{p}}{d t^{p}}=a_{1}\left(u_{1}+u_{2}\right)+\kappa_{1}\left[u_{1}(t)-u_{1}(t-\varrho)\right], \\
\frac{d u_{2}^{p}}{d t^{p}}=-u_{2}-a_{1} u_{1} u_{3}+\kappa_{2}\left[u_{2}(t)-u_{2}(t-\varrho)\right] \\
\frac{d u_{3}^{p}}{d t^{p}}=a_{2}+a_{1} u_{1} u_{2} .
\end{array}\right.
$$

The linear equation of (3.2) near the equilibrium point $E_{1}$ takes the form

$$
\left\{\begin{array}{l}
\left.\frac{d u_{1}^{p}}{d t^{p}}=\left(a_{1}+\kappa_{1}\right) u_{1}+a_{1} u_{2}-\kappa_{1} u_{1}(t-\varrho)\right], \\
\frac{d u_{2}^{p}}{d t^{p}}=-u_{1}+\left(\kappa_{2}-1\right) u_{2}+\sqrt{a_{1} a_{2}} u_{3}-\kappa_{2} u_{2}(t-\varrho), \\
\frac{d u_{3}^{p}}{d t^{p}}=\sqrt{a_{1} a_{2}} u_{1}-\sqrt{a_{1} a_{2}} u_{2} .
\end{array}\right.
$$

The corresponding characteristic equation of (3.3) is given by

$$
\operatorname{det}\left[\begin{array}{ccc}
s^{p}-\left(a_{1}+\kappa_{1}\right)+\kappa_{1} e^{-s \varrho} & -a_{1} & 0 \\
1 & s^{p}-\left(\kappa_{2}-1\right)+\kappa_{2} e^{-s \varrho} & -\sqrt{a_{1} a_{2}} \\
-\sqrt{a_{1} a_{2}} & \sqrt{a_{1} a_{2}} & s^{p}
\end{array}\right],
$$

which leads to

$$
\mathcal{A}_{1}(s)+\mathcal{A}_{2}(s) e^{-s \varrho}+\mathcal{A}_{3}(s) e^{-2 s \varrho}=0
$$

where

$$
\begin{aligned}
\mathcal{A}_{1}(s)= & s^{3 p}-\left(\kappa_{1}-1+a_{1}+\kappa_{2}\right) s^{2 p}+\left[a_{1} a_{2}-\kappa_{1}-\left(a_{1}+\kappa_{1}\right)\left(\kappa_{2}-1\right)\right] s^{p} \\
& +a_{1} a_{2} \kappa_{1}, \\
\mathcal{A}_{2}(s)= & \left(\kappa_{1}+\kappa_{2}\right) s^{2 p}-\left[\kappa_{2}\left(a_{1}+\kappa_{1}\right)+\kappa_{1}\left(\kappa_{2}-1\right)\right] s^{p}+a_{1} a_{2} \kappa_{1}, \\
\mathcal{A}_{3}(s)= & \kappa_{1} \kappa_{2} s^{p} .
\end{aligned}
$$


It follows from (3.5) that

$$
\mathcal{A}_{1}(s) e^{s \varrho}+\mathcal{A}_{2}(s)+\mathcal{A}_{3}(s) e^{-s \varrho}=0
$$

Let $s=i \phi=\phi\left(\cos \frac{\pi}{2}+i \sin \frac{\pi}{2}\right)$ be a root of (3.6). Then

$$
\left\{\begin{array}{l}
\mathcal{H}_{1}(\phi) \cos \phi \sigma+\mathcal{H}_{2}(\phi) \sin \phi \sigma=\mathcal{H}_{3}(\phi), \\
\mathcal{G}_{1}(\phi) \cos \phi \sigma+\mathcal{G}_{2}(\phi) \sin \phi \sigma=\mathcal{G}_{3}(\phi),
\end{array}\right.
$$

where

$$
\begin{aligned}
\mathcal{H}_{1}(\phi)= & \phi^{3 p} \cos \frac{3 p \pi}{2}-\left(\kappa_{1}-1+a_{1}+\kappa_{2}\right) \phi^{2 p} \cos p \pi+\left[a_{1} a_{2}-\kappa_{1}\right. \\
& \left.-\left(a_{1}+\kappa_{1}\right)\left(\kappa_{2}-1\right)+\kappa_{1} \kappa_{2}\right] \phi^{p} \cos \frac{p \pi}{2}+a_{1} a_{2} \kappa_{1}, \\
\mathcal{H}_{2}(\phi)= & -\phi^{3 p} \sin \frac{3 p \pi}{2}+\left(\kappa_{1}-1+a_{1}+\kappa_{2}\right) \phi^{2 p} \sin p \pi-\left[a_{1} a_{2}-\kappa_{1}\right. \\
& \left.-\left(a_{1}+\kappa_{1}\right)\left(\kappa_{2}-1\right)+\kappa_{1} \kappa_{2}\right] \phi^{p} \sin \frac{p \pi}{2} \\
\mathcal{H}_{3}(\phi)= & -\left(\kappa_{1}+\kappa_{2}\right) \phi^{2 p} \cos p \pi+\left[\kappa_{2}\left(a_{1}+\kappa_{1}\right)+\kappa_{1}\left(\kappa_{2}-1\right)\right] \phi^{p} \cos \frac{p \pi}{2}-a_{1} a_{2} \kappa_{1}, \\
\mathcal{G}_{1}(\phi)= & \phi^{3 p} \sin \frac{3 p \pi}{2}-\left(\kappa_{1}-1+a_{1}+\kappa_{2}\right) \phi^{2 p} \cos p \pi+\left[a_{1} a_{2}-\kappa_{1}\right. \\
& \left.-\left(a_{1}+\kappa_{1}\right)\left(\kappa_{2}-1\right)+\kappa_{1} \kappa_{2}\right] \phi^{p} \sin \frac{p \pi}{2}, \\
& \left.-\left(a_{1}+\kappa_{1}\right)\left(\kappa_{2}-1\right)-\kappa_{1} \kappa_{2}\right] \phi^{p} \cos \frac{p \pi}{2}+a_{1} a_{2}\left(a_{1}+\kappa_{1}\right), \\
\mathcal{G}_{2}(\phi)= & \phi^{3 p} \cos \frac{3 p \pi}{2}-\left(\kappa_{1}-1+a_{1}+\kappa_{2}\right) \phi^{2 p} \cos p \pi+\left[a_{1} a_{2}-\kappa_{1}\right. \\
\mathcal{G}_{3}(\phi)= & -\left(\kappa_{1}+\kappa_{2}\right) \phi^{2 p} \sin p \pi+\left[\kappa_{2}\left(a_{1}+\kappa_{1}\right)+\kappa_{1}\left(\kappa_{2}-1\right)\right] \phi^{p} \sin \frac{p \pi}{2} .
\end{aligned}
$$

Let

$$
\left\{\begin{array}{l}
\alpha_{1}=\cos \frac{3 p \pi}{2}, \quad \alpha_{2}=-\left(\kappa_{1}-1+a_{1}+\kappa_{2}\right) \cos p \pi, \\
\alpha_{3}=\left[a_{1} a_{2}-\kappa_{1}-\left(a_{1}+\kappa_{1}\right)\left(\kappa_{2}-1\right)+\kappa_{1} \kappa_{2}\right] \cos \frac{p \pi}{2}, \\
\alpha_{4}=a_{1} a_{2} \kappa_{1}, \quad \alpha_{5}=-\sin \frac{3 p \pi}{2}, \quad \alpha_{6}=\left(\kappa_{1}-1+a_{1}+\kappa_{2}\right) \sin p \pi, \\
\alpha_{7}=-\left[a_{1} a_{2}-\kappa_{1}-\left(a_{1}+\kappa_{1}\right)\left(\kappa_{2}-1\right)+\kappa_{1} \kappa_{2}\right] \sin \frac{p \pi}{2}, \\
\alpha_{8}=-\left(\kappa_{1}+\kappa_{2}\right) \cos p \pi, \quad \alpha_{9}=\left[\kappa_{2}\left(a_{1}+\kappa_{1}\right)+\kappa_{1}\left(\kappa_{2}-1\right)\right] \cos \frac{p \pi}{2}, \\
\alpha_{10}=-a_{1} a_{2} \kappa_{1}, \quad \alpha_{11}=\sin \frac{3 p \pi}{2}, \quad \alpha_{12}=-\left(\kappa_{1}-1+a_{1}+\kappa_{2}\right) \cos p \pi \\
\alpha_{13}=\left[a_{1} a_{2}-\kappa_{1}-\left(a_{1}+\kappa_{1}\right)\left(\kappa_{2}-1\right)+\kappa_{1} \kappa_{2}\right] \sin \frac{p \pi}{2}, \\
\alpha_{15}=-\left(\kappa_{1}-1+a_{1}+\kappa_{2}\right) \cos p \pi, \\
\alpha_{16}=\left[a_{1} a_{2}-\kappa_{1}-\left(a_{1}+\kappa_{1}\right)\left(\kappa_{2}-1\right)-\kappa_{1} \kappa_{2}\right] \cos \frac{p \pi}{2}, \\
\alpha_{17}=a_{1} a_{2}\left(a_{1}+\kappa_{1}\right), \quad \alpha_{18}=-\left(\kappa_{1}+\kappa_{2}\right) \sin p \pi, \\
\alpha_{19}=\left[\kappa_{2}\left(a_{1}+\kappa_{1}\right)+\kappa_{1}\left(\kappa_{2}-1\right)\right] \sin \frac{p \pi}{2} .
\end{array}\right.
$$


Then

$$
\left\{\begin{array}{l}
\mathcal{H}_{1}(\phi)=\alpha_{1} \phi^{3 p}+\alpha_{2} \phi^{2 p}+\alpha_{3} \phi^{p}+\alpha_{4}, \\
\mathcal{H}_{2}(\phi)=\alpha_{5} \phi^{3 p}+\alpha_{6} \phi^{2 p}+\alpha_{7} \phi^{p}, \\
\mathcal{H}_{3}(\phi)=\alpha_{8} \phi^{2 p}+\alpha_{9} \phi^{p}+\alpha_{10}, \\
\mathcal{G}_{1}(\phi)=\alpha_{11} \phi^{3 p}+\alpha_{12} \phi^{2 p}+\alpha_{13} \phi^{p}, \\
\mathcal{G}_{2}(\phi)=\alpha_{14} \phi^{3 p}+\alpha_{15} \phi^{2 p}+\alpha_{16} \phi^{p}+\alpha_{17}, \\
\mathcal{G}_{3}(\phi)=\alpha_{18} \phi^{2 p}+\alpha_{19} \phi^{p} .
\end{array}\right.
$$

By (3.7), one has

$$
\left\{\begin{array}{l}
\cos \phi \sigma=\frac{\mathcal{H}_{3}(\phi) \mathcal{G}_{2}(\phi)-\mathcal{G}_{3}(\phi) \mathcal{H}_{2}(\phi)}{\mathcal{H}_{1}(\phi) \mathcal{G}_{2}(\phi)-\mathcal{G}_{1}(\phi) \mathcal{H}_{2}(\phi)} \\
\sin \varphi \sigma=\frac{\mathcal{H}_{1}(\phi) \mathcal{G}_{3}(\phi)-\mathcal{G}_{1}(\phi) \mathcal{H}_{3}(\phi)}{\mathcal{H}_{1}(\phi) \mathcal{G}_{2}(\phi)-\mathcal{G}_{1}(\phi) \mathcal{H}_{2}(\phi)}
\end{array}\right.
$$

In view of the equation $\cos ^{2} \phi \sigma+\sin ^{2} \phi \sigma=1$, one has

$$
\begin{aligned}
& {\left[\mathcal{H}_{3}(\phi) \mathcal{G}_{2}(\phi)-\mathcal{G}_{3}(\phi) \mathcal{H}_{2}(\phi)\right]^{2}+\left[\mathcal{H}_{1}(\phi) \mathcal{G}_{3}(\phi)-\mathcal{G}_{1}(\phi) \mathcal{H}_{3}(\phi)\right]^{2}} \\
& \quad=\left[\mathcal{H}_{1}(\phi) \mathcal{G}_{2}(\phi)-\mathcal{G}_{1}(\phi) \mathcal{H}_{2}(\phi)\right]^{2}
\end{aligned}
$$

Since

$$
\begin{aligned}
& {\left[\mathcal{H}_{3}(\phi) \mathcal{G}_{2}(\phi)-\mathcal{G}_{3}(\phi) \mathcal{H}_{2}(\phi)\right]^{2}=\left(l_{1} \phi^{5 p}+l_{2} \phi^{4 p}+l_{3} \phi^{3 p}+l_{4} \phi^{2 p}+l_{5} \phi^{p}+l_{6}\right)^{2}} \\
& {\left[\mathcal{H}_{1}(\phi) \mathcal{G}_{3}(\phi)-\mathcal{G}_{1}(\phi) \mathcal{H}_{3}(\phi)\right]^{2}=\left(l_{7} \phi^{5 p}+l_{8} \phi^{4 p}+l_{9} \phi^{3 p}+l_{10} \phi^{2 p}+l_{11} \phi^{p}\right)^{2}} \\
& {\left[\mathcal{H}_{1}(\phi) \mathcal{G}_{2}(\phi)-\mathcal{G}_{1}(\phi) \mathcal{H}_{2}(\phi)\right]^{2}} \\
& \quad=\left(l_{12} \phi^{6 p}+l_{13} \phi^{5 p}+l_{14} \phi^{4 p}+l_{15} \phi^{3 p}+l_{16} \phi^{2 p}+l_{17} \phi^{p}+l_{18}\right)^{2}
\end{aligned}
$$

where

$$
\left\{\begin{array}{l}
l_{1}=\alpha_{8} \alpha_{14}-\alpha_{5} \alpha_{18}, \quad l_{2}=\alpha_{8} \alpha_{15}+\alpha_{9} \alpha_{14}-\alpha_{6} \alpha_{18}-\alpha_{5} \alpha_{19}, \\
l_{3}=\alpha_{8} \alpha_{16}+\alpha_{9} \alpha_{15}+\alpha_{10} \alpha_{14}-\alpha_{7} \alpha_{18}-\alpha_{6} \alpha_{19}, \\
l_{4}=\alpha_{8} \alpha_{17}+\alpha_{9} \alpha_{16}+\alpha_{10} \alpha_{15}-\alpha_{7} \alpha_{19}, \quad l_{5}=\alpha_{10} \alpha_{16}, \quad l_{6}=\alpha_{10} \alpha_{17}, \\
l_{7}=\alpha_{1} \alpha_{18}-\alpha_{8} \alpha_{11}, \quad l_{8}=\alpha_{1} \alpha_{19}+\alpha_{2} \alpha_{18}-\alpha_{9} \alpha_{11}-\alpha_{8} \alpha_{12}, \\
l_{9}=\alpha_{2} \alpha_{19}+\alpha_{3} \alpha_{18}-\alpha_{10} \alpha_{11}-\alpha_{9} \alpha_{12}-\alpha_{8} \alpha_{13}, \\
l_{10}=\alpha_{3} \alpha_{19}+\alpha_{4} \alpha_{18}-\alpha_{10} \alpha_{12}-\alpha_{9} \alpha_{13}, \quad l_{11}=\alpha_{4} \alpha_{19}-\alpha_{10} \alpha_{13}, \\
l_{12}=\alpha_{1} \alpha_{14}-\alpha_{3} \alpha_{11}, \quad l_{13}=\alpha_{1} \alpha_{15}+\alpha_{2} \alpha_{14}-\alpha_{6} \alpha_{11}-\alpha_{3} \alpha_{12}, \\
l_{14}=\alpha_{1} \alpha_{16}+\alpha_{2} \alpha_{15}+\alpha_{3} \alpha_{14}-\alpha_{7} \alpha_{11}-\alpha_{6} \alpha_{12}-\alpha_{3} \alpha_{13}, \\
l_{15}=\alpha_{1} \alpha_{17}+\alpha_{2} \alpha_{16}+\alpha_{3} \alpha_{15}+\alpha_{4} \alpha_{14}-\alpha_{7} \alpha_{12}-\alpha_{7} \alpha_{13} \\
l_{16}=\alpha_{2} \alpha_{17}+\alpha_{3} \alpha_{16}+\alpha_{4} \alpha_{15}-\alpha_{7} \alpha_{13}, \\
l_{17}=\alpha_{3} \alpha_{17}+\alpha_{4} \alpha_{16}, \quad l_{18}=\alpha_{4} \alpha_{17},
\end{array}\right.
$$


we have

$$
\begin{gathered}
b_{1} \phi^{12 p}+b_{2} \phi^{11 p}+b_{3} \phi^{10 p}+b_{4} \phi^{9 p}+b_{5} \phi^{8 p}+b_{6} \phi^{7 p}+b_{7} \phi^{6 p} \\
+b_{8} \phi^{5 p}+b_{9} \phi^{4 p}+b_{10} \phi^{3 p}+b_{11} \phi^{2 p}+b_{12} \phi^{p}+b_{13}=0
\end{gathered}
$$

where

$$
\left\{\begin{array}{l}
b_{1}=l_{12}^{2}, \quad b_{2}=2 l_{12} l_{13}, \quad b_{3}=l_{13}^{2}+2 l_{12} l_{14}-l_{7}^{2}-l_{1}^{2}, \\
b_{4}=2\left(l_{12} l_{15}+l_{13} l_{14}-l_{7} l_{8}-l_{1} l_{2}\right), \\
b_{5}=l_{14}^{2}+2 l_{12} l_{16}+2 l_{13} l_{15}-l_{8}^{2}-2 l_{7} l_{8}-l_{2}^{2}-2 l_{1} l_{3}, \\
b_{6}=2\left[l_{12} l_{17}+l_{13} l_{16}+l_{14} l_{15}-l_{8} l_{9}-l_{7} l_{10}-l_{1} l_{4}-l_{2} l_{3}\right], \\
b_{7}=l_{15}^{2}+2 l_{12} l_{18}+2 l_{14} l_{16}+2 l_{13} l_{17}-l_{9}^{2}-2 l_{7} l_{11}-2 l_{8} l_{10}-l_{3}^{2}-2 l_{1} l_{5}-2 l_{2} l_{4}, \\
b_{8}=2\left[l_{13} l_{18}+l_{14} l_{17}+l_{15} l_{16}-l_{8} l_{11}-l_{9} l_{10}-l_{1} l_{6}-l_{2} l_{5}-l_{3} l_{4}\right], \\
b_{9}=l_{16}^{2}+2 l_{14} l_{18}+2 l_{15} l_{17}-l_{10}^{2}-2 l_{9} l_{11}-l_{4}^{2}-2 l_{2} l_{6}-2 l_{3} l_{5}, \\
b_{10}=2\left[l_{15} l_{18}+l_{16} l_{17}-l_{10} l_{11}-l_{3} l_{6}-l_{4} l_{5}\right], \\
b_{11}=l_{17}^{2}+2 l_{16} l_{18}-l_{5}^{2}-2 l_{4} l_{6}, \quad b_{12}=2\left(l_{17} l_{18}-2 l_{5} l_{6}\right), \quad b_{13}=l_{18}^{2} .
\end{array}\right.
$$

Denote

$$
\begin{aligned}
\chi(\phi)= & b_{1} \phi^{12 p}+b_{2} \phi^{11 p}+b_{3} \phi^{10 p}+b_{4} \phi^{9 p}+b_{5} \phi^{8 p}+b_{6} \phi^{7 p}+b_{7} \phi^{6 p} \\
& +b_{8} \phi^{5 p}+b_{9} \phi^{4 p}+b_{10} \phi^{3 p}+b_{11} \phi^{2 p}+b_{12} \phi^{p}+b_{13}
\end{aligned}
$$

and

$$
\begin{aligned}
\rho(\mu)= & b_{1} \mu^{12}+b_{2} \mu^{11}+b_{3} \mu^{10}+b_{4} \mu^{9}+b_{5} \mu^{8}+b_{6} \mu^{7}+b_{7} \mu^{6} \\
& +b_{8} \mu^{5}+b_{9} \mu^{4}+b_{10} \mu^{3}+b_{11} \mu^{2}+b_{12} \mu+b_{13} .
\end{aligned}
$$

The following assumption is given:

$(\mathcal{A} 1) \kappa_{1} \neq 0$.

Lemma 3.1 For (3.5), the following conclusions are true:

(i) If $b_{i}>0(i=1,2,3, \ldots, 12)$, then (3.5) possesses no root with zero real parts.

(ii) If there exists a positive constant $\mu_{0}$ such that $\rho\left(\mu_{0}\right)<0$, then (3.5) possesses at least two pairs of purely imaginary roots.

Proof We will prove the two cases, respectively.

(i) By (3.15), one gets

$$
\begin{aligned}
\frac{d \chi(\phi)}{d \phi}= & 12 p b_{1} \phi^{12 p-1}+11 p b_{2} \phi^{11 p-1}+10 p b_{3} \phi^{10 p-1}+9 p b_{4} \phi^{9 p-1}+8 p b_{5} \phi^{8 p-1} \\
& +7 p b_{6} \phi^{7 p-1}+6 p b_{7} \phi^{6 p-1}+5 p b_{8} \phi^{5 p-1}+4 p b_{9} \phi^{4 p-1}+3 p b_{10} \phi^{3 p-1} \\
& +2 p b_{11} \phi^{2 p-1}+p b_{12} \phi^{p-1} .
\end{aligned}
$$


Since $b_{i}>0(i=1,2,3, \ldots, 13)$, we have $\frac{d \chi(\phi)}{d \phi}>0 \forall \phi>0$. In view of $\chi(0)=b_{13}>0$, we know that (3.15) possesses no positive real root. In view of $(\mathcal{A} 1)$, we can conclude that $s=0$ is not the root of (3.5). The proof of (i) is completed.

(ii) Because $\rho(0)=b_{13}>0, \rho\left(\varepsilon_{0}\right)<0\left(\varepsilon_{0}>0\right)$ and $\lim _{\nu \rightarrow+\infty} \frac{\rho(v)}{d v}=+\infty$, one can know that $\exists \varepsilon_{01} \in\left(0, \varepsilon_{0}\right)$ and $\varepsilon_{02} \in\left(\varepsilon_{0},+\infty\right)$ such that $\rho\left(\varepsilon_{01}\right)=\rho\left(\varepsilon_{02}\right)=0$, which implies that (3.13) possesses at least two positive real roots. Then (3.5) possesses at least two pairs of purely imaginary roots. The proof of (ii) is finished.

Without loss of generality, assume that (3.13) has six positive real roots identified by $\phi_{l}$ $(l=1,2, \ldots, 13)$. By $(3.10)$, one gets

$$
\varrho_{l}^{k}=\frac{1}{\phi_{l}}\left[\arccos \left(\frac{\mathcal{H}_{3}\left(\phi_{l}\right) \mathcal{G}_{2}\left(\phi_{l}\right)-\mathcal{G}_{3}\left(\phi_{l}\right) \mathcal{H}_{2}\left(\phi_{l}\right)}{\mathcal{H}_{1}\left(\phi_{l}\right) G_{2}\left(\phi_{l}\right)-\mathcal{G}_{1}\left(\phi_{l}\right) \mathcal{H}_{2}\left(\phi_{l}\right)}\right)+2 k \pi\right]
$$

where $k=0,1,2, \ldots, l=1,2, \ldots, 13$. Then $\pm i \phi_{l}$ is a pair of purely imaginary roots of (3.5) when $\varrho=\varrho_{l}^{k}$. Let

$$
\varrho_{0}=\min _{l=1,2, \ldots, 13}\left\{\varrho_{l}^{0}\right\}, \quad \phi_{0}=\left.\phi\right|_{\varrho=\varrho_{0}} .
$$

Now the following assumption is made:

$(\mathcal{A} 2) \mathcal{M}_{1} \mathcal{N}_{1}+\mathcal{M}_{2} \mathcal{N}_{2}>0$, where

$$
\begin{aligned}
\mathcal{M}_{1}= & \kappa_{1} \kappa_{2} \phi_{0}^{p}\left(\cos \phi_{0} \varrho_{0} \cos \frac{p \pi}{2}+\sin \phi_{0} \varrho_{0} \sin \frac{p \pi}{2}\right) \\
& +\phi_{0} \sin \phi_{0} \varrho_{0}\left[\phi_{0}^{3 p} \cos \frac{3 p \pi}{2}-\left(\kappa_{1}-1+a_{1}+\kappa_{2}\right) \phi_{0}^{2 p} \cos p \pi\right. \\
& \left.+\left(a_{1} a_{2}-\kappa_{1}-\left(a_{1}+\kappa_{1}\right)\left(\kappa_{2}-1\right)\right) \phi_{0}^{p} \cos \frac{p \pi}{2}+a_{1} a_{2} \kappa_{1}\right] \\
& +\phi_{0} \cos \phi_{0} \varrho_{0}\left[\phi_{0}^{3 p} \sin \frac{3 p \pi}{2}-\left(\kappa_{1}-1+a_{1}+\kappa_{2}\right) \phi_{0}^{2 p} \sin p \pi\right. \\
& \left.+\left(a_{1} a_{2}-\kappa_{1}-\left(a_{1}+\kappa_{1}\right)\left(\kappa_{2}-1\right)\right) \phi_{0}^{p} \sin \frac{p \pi}{2}\right] \\
\mathcal{M}_{2}= & \kappa_{1} \kappa_{2} \phi_{0}^{p}\left(\cos \phi_{0} \varrho_{0} \sin \frac{p \pi}{2}-\sin \phi_{0} \varrho_{0} \cos \frac{p \pi}{2}\right) \\
& +\phi_{0} \sin \phi_{0} \varrho_{0}\left[\phi_{0}^{3 p} \sin \frac{3 p \pi}{2}-\left(\kappa_{1}-1+a_{1}+\kappa_{2}\right) \phi_{0}^{2 p} \sin p \pi\right. \\
& \left.+\left(a_{1} a_{2}-\kappa_{1}-\left(a_{1}+\kappa_{1}\right)\left(\kappa_{2}-1\right)\right) \phi_{0}^{p} \sin \frac{p \pi}{2}\right] \\
& +\phi_{0} \cos \phi_{0} \varrho_{0}\left[\phi_{0}^{3 p} \cos \frac{3 p \pi}{2}-\left(\kappa_{1}-1+a_{1}+\kappa_{2}\right) \phi_{0}^{2 p} \cos p \pi\right. \\
& \left.+\left(a_{1} a_{2}-\kappa_{1}-\left(a_{1}+\kappa_{1}\right)\left(\kappa_{2}-1\right)\right) \phi_{0}^{p} \cos \frac{p \pi}{2}+a_{1} a_{2} \kappa_{1}\right] \\
\mathcal{N}_{1}=[ & 3 p \phi_{0}^{3 p} \cos \frac{3 p \pi}{2}-2 p\left(\kappa_{1}-1+a_{1}+\kappa_{2}\right) \phi_{0}^{2 p-1} \cos \frac{(2 p-1) \pi}{2}
\end{aligned}
$$




$$
\begin{aligned}
& \left.+p\left(a_{1} a_{2}-\kappa_{1}-\left(a_{1}+\kappa_{1}\right)\left(\kappa_{2}-1\right)\right) \phi_{0}^{p-1} \cos \frac{(p-1) \pi}{2}\right] \cos \phi_{0} \varrho_{0} \\
& -\sin \phi_{0} \varrho_{0}\left[3 p \phi_{0}^{3 p} \sin \frac{3 p \pi}{2}-2 p\left(\kappa_{1}-1+a_{1}+\kappa_{2}\right) \phi_{0}^{2 p-1} \sin \frac{(2 p-1) \pi}{2}\right. \\
& \left.+p\left(a_{1} a_{2}-\kappa_{1}-\left(a_{1}+\kappa_{1}\right)\left(\kappa_{2}-1\right)\right) \phi_{0}^{p-1} \sin \frac{(p-1) \pi}{2}\right] \\
& +\varrho_{0} \cos \phi_{0} \varrho_{0}\left[\phi_{0}^{3 p} \cos \frac{3 p \pi}{2}-\left(\kappa_{1}-1+a_{1}+\kappa_{2}\right) \phi_{0}^{2 p} \cos \frac{2 p \pi}{2}\right. \\
& \left.+\left(a_{1} a_{2}-\kappa_{1}-\left(a_{1}+\kappa_{1}\right)\left(\kappa_{2}-1\right)\right) \phi_{0}^{p} \cos \frac{p \pi}{2}+a_{1} a_{2} \kappa_{1}\right] \\
& -\varrho_{0} \sin \phi_{0} \varrho_{0}\left[\phi_{0}^{3 p} \sin \frac{3 p \pi}{2}-\left(\kappa_{1}-1+a_{1}+\kappa_{2}\right) \phi_{0}^{2 p} \sin \frac{2 p \pi}{2}\right. \\
& \left.+\left(a_{1} a_{2}-\kappa_{1}-\left(a_{1}+\kappa_{1}\right)\left(\kappa_{2}-1\right)\right) \phi_{0}^{p} \sin \frac{p \pi}{2}\right]+2 p\left(\kappa_{1}+\kappa_{2}\right) \phi_{0}^{2 p-1} \cos \frac{(2 p-1) \pi}{2} \\
& -p\left[\kappa_{2}\left(a-1+a_{2}\right)-\kappa_{1}\left(\kappa_{2}-1\right)\right] \phi_{0}^{p-1} \cos \frac{(p-1) \pi}{2} \\
& +p \kappa_{1} \kappa_{2} \phi_{0}^{p-1} \cos \frac{(p-1) \pi}{2} \cos \phi_{0} \varrho_{0}-p \kappa_{1} \kappa_{2} \phi_{0}^{p-1} \sin \frac{(p-1) \pi}{2} \sin \phi_{0} \varrho_{0} \\
& +\varrho_{0} \kappa_{1} \kappa_{2} \phi_{0}^{p} \cos \frac{p \pi}{2} \cos \phi_{0} \varrho_{0}+\varrho_{0} \kappa_{1} \kappa_{2} \phi_{0}^{p} \sin \frac{p \pi}{2} \sin \phi_{0} \varrho_{0} \\
& \mathcal{N}_{2}=\left[3 p \phi_{0}^{3 p} \cos \frac{3 p \pi}{2}-2 p\left(\kappa_{1}-1+a_{1}+\kappa_{2}\right) \phi_{0}^{2 p-1} \cos \frac{(2 p-1) \pi}{2}\right. \\
& \left.+p\left(a_{1} a_{2}-\kappa_{1}-\left(a_{1}+\kappa_{1}\right)\left(\kappa_{2}-1\right)\right) \phi_{0}^{p-1} \cos \frac{(p-1) \pi}{2}\right] \sin \phi_{0} \varrho_{0} \\
& -\cos \phi_{0} \varrho_{0}\left[3 p \phi_{0}^{3 p} \sin \frac{3 p \pi}{2}-2 p\left(\kappa_{1}-1+a_{1}+\kappa_{2}\right) \phi_{0}^{2 p-1} \sin \frac{(2 p-1) \pi}{2}\right. \\
& \left.+p\left(a_{1} a_{2}-\kappa_{1}-\left(a_{1}+\kappa_{1}\right)\left(\kappa_{2}-1\right)\right) \phi_{0}^{p-1} \sin \frac{(p-1) \pi}{2}\right] \\
& +\varrho_{0} \sin \phi_{0} \varrho_{0}\left[\phi_{0}^{3 p} \cos \frac{3 p \pi}{2}-\left(\kappa_{1}-1+a_{1}+\kappa_{2}\right) \phi_{0}^{2 p} \cos \frac{2 p \pi}{2}\right. \\
& \left.+\left(a_{1} a_{2}-\kappa_{1}-\left(a_{1}+\kappa_{1}\right)\left(\kappa_{2}-1\right)\right) \phi_{0}^{p} \cos \frac{p \pi}{2}+a_{1} a_{2} \kappa_{1}\right] \\
& +\varrho_{0} \cos \phi_{0} \varrho_{0}\left[\phi_{0}^{3 p} \sin \frac{3 p \pi}{2}-\left(\kappa_{1}-1+a_{1}+\kappa_{2}\right) \phi_{0}^{2 p} \sin \frac{2 p \pi}{2}\right. \\
& \left.+\left(a_{1} a_{2}-\kappa_{1}-\left(a_{1}+\kappa_{1}\right)\left(\kappa_{2}-1\right)\right) \phi_{0}^{p} \sin \frac{p \pi}{2}\right]+2 p\left(\kappa_{1}+\kappa_{2}\right) \phi_{0}^{2 p-1} \sin \frac{(2 p-1) \pi}{2} \\
& -p\left[\kappa_{2}\left(a-1+a_{2}\right)-\kappa_{1}\left(\kappa_{2}-1\right)\right] \phi_{0}^{p-1} \sin \frac{(p-1) \pi}{2} \\
& +p \kappa_{1} \kappa_{2} \phi_{0}^{p-1} \cos \frac{(p-1) \pi}{2} \sin \phi_{0} \varrho_{0}+p \kappa_{1} \kappa_{2} \phi_{0}^{p-1} \sin \frac{(p-1) \pi}{2} \cos \phi_{0} \varrho_{0} \\
& -\varrho_{0} \kappa_{1} \kappa_{2} \phi_{0}^{p} \cos \frac{p \pi}{2} \sin \phi_{0} \varrho_{0}+\varrho_{0} \kappa_{1} \kappa_{2} \phi_{0}^{p} \sin \frac{p \pi}{2} \cos \phi_{0} \varrho_{0} .
\end{aligned}
$$

Lemma 3.2 Suppose that $s(\varrho)=v(\varrho)+i \phi(\varrho)$ is the root of $(3.5)$ at $\varrho=\varrho_{0}$ satisfying $v\left(\varrho_{0}\right)=0$, $\phi\left(\varrho_{0}\right)=\phi_{0}$, then $\left.\operatorname{Re}\left[\frac{d s}{d \varrho}\right]\right|_{\varrho=\varrho_{0}, \phi=\phi_{0}}>0$. 
Proof According to (3.6), one gets

$$
\frac{d s}{d \varrho}=\frac{\mathcal{B}_{1}(\varrho)}{\mathcal{B}_{2}(\varrho)}
$$

where

$$
\begin{aligned}
\mathcal{B}_{1}(\varrho)= & e^{-s \varrho} \kappa_{1} \kappa_{2} s^{p}-s e^{s \varrho}\left[s^{3 p}-\left(\kappa_{1}-1+a_{1}+\kappa_{2}\right) s^{2 p}\right. \\
& \left.+\left(a_{1} a_{2}-\kappa_{1}-\left(a_{1}+\kappa_{1}\right)\left(\kappa_{2}-1\right)\right) s^{p}+a_{1} a_{2} \kappa_{1}\right] \\
\mathcal{B}_{2}(\varrho)= & \left\{3 p s^{3 p-1}-2 p\left(\kappa_{1}-1+a_{1}+\kappa_{2}\right) s^{2 p-1}+p\left[a_{1} a_{2}-\kappa_{1}-\left(a_{1}+\kappa_{1}\right)\left(\kappa_{2}-1\right)\right] s^{p-1}\right\} e^{s \varrho} \\
& +e^{s \varrho} \varrho\left[s^{3 p}-\left(\kappa_{1}-1+a_{1}+\kappa_{2}\right) s^{2 p}+\left(a_{1} a_{2}-\kappa_{1}-\left(a_{1}+\kappa_{1}\right)\left(\kappa_{2}-1\right)\right) s^{p}+a_{1} a_{2} \kappa_{1}\right] \\
& +2 p\left(\kappa_{1}+\kappa_{2}\right) s^{2 p-1}-p\left[\kappa_{2}\left(a_{1}+a_{2}\right)-\kappa_{1}\left(\kappa_{2}-1\right)\right] s^{p-1} \\
& +p \kappa_{1} \kappa_{2} s^{p-1} e^{-s \varrho}-e^{-s \varrho} \varrho \kappa_{1} \kappa_{2} s^{p} .
\end{aligned}
$$

Then

$$
\operatorname{Re}\left\{\frac{d s}{d \sigma}\right\}=\operatorname{Re}\left\{\frac{\mathcal{B}_{1}(\varrho)}{\mathcal{B}_{2}(\varrho)}\right\}
$$

Hence

$$
\left.\operatorname{Re}\left\{\frac{d s}{d \sigma}\right\}\right|_{\varrho=\varrho_{0}, \phi=\phi_{0}}=\left.\operatorname{Re}\left\{\frac{B_{1}(\varrho)}{B_{2}(\varrho)}\right\}\right|_{\varrho=\varrho_{0}, \phi=\phi_{0}}=\frac{\mathcal{M}_{1} \mathcal{N}_{1}+\mathcal{M}_{2} \mathcal{N}_{2}}{\mathcal{N}_{1}^{2}+\mathcal{N}_{2}^{2}}
$$

In terms of $(\mathcal{A} 3)$, one has

$$
\left.\operatorname{Re}\left\{\left[\frac{d s}{d \varrho}\right]^{-1}\right\}\right|_{\varrho=\varrho_{0}, \phi=\phi_{0}}>0
$$

The proof of Lemma 3.2 is finished.

Next we give an assumption as follows:

$(\mathcal{A} 3) \kappa_{1}>0,\left(1-a_{1}\right)\left(a_{1} a_{2}-2 \kappa_{1} \kappa_{2}-2 a_{1} \kappa_{2}+a_{1}+\kappa_{1}\right)>2 a_{1} a_{2} \kappa_{1}$.

Lemma 3.3 If $\varrho=0$ and $(\mathcal{A} 3)$ hold true, then system (3.2) is asymptotically stable.

Proof If $\varrho=0$, then (3.5) takes the form

$$
\lambda^{3}+\left(1-a_{1}\right) \lambda^{2}+\left(a_{1} a_{2}-2 \kappa_{1} \kappa_{2}-2 a_{1} \kappa_{2}+a_{1}+\kappa_{1}\right) \lambda+2 a_{1} a_{2} \kappa_{1}=0
$$

It follows from $(\mathcal{A} 3)$ that all the roots $\lambda_{i}$ of (3.20) satisfy $\left|\arg \left(\lambda_{i}\right)\right|>\frac{p \pi}{2}(i=1,2)$. By Lemma 2.1 , we know that system (3.2) with $\varrho=0$ is asymptotically stable. The proof of Lemma 3.3 is finished.

According to the analysis above and Lemmas 3.2 and 3.3, one has the following theorem. 
Theorem 3.1 For system (3.2), assume that $(\mathcal{A} 1)-(\mathcal{A} 3)$ are satisfied, then the equilibrium point $E_{1}$ is globally asymptotically stable for $\varrho \in\left[0, \varrho_{0}\right)$ and system (3.2) undergoes a Hopf bifurcation near the equilibrium point $E_{1}$ when $\varrho=\varrho_{0}$.

Remark 3.1 In $[1-3,53]$, the authors studied the Hopf bifurcation and chaotic behavior of integer-order finance systems. In this paper, we investigate the chaos control of fractional-order delayed finance systems. All the obtained results and analysis methods $[1-3,53]$ cannot be applied to (3.2) to obtain the stability and the existence of Hopf bifurcation for (3.2). For these reasons, the fruits of our research about the chaos control for (1.2) are completely innovative and are an important supplement to some previous research results.

Remark 3.2 Xu and Zhang [54] focused on the chaos control of the Qi system by linear time-delay feedback control. They do not involve fractional-order models. From this viewpoint, the results of this article also supplement the research of Xu and Zhang [54].

\section{An example}

Consider the fractional-order finance model:

$$
\left\{\begin{array}{l}
\frac{d^{0.8} u_{1}}{d 0^{0.8}}=1.79\left(u_{1}+u_{2}\right)+\kappa_{1}\left[u_{1}(t)-u_{1}(t-\varrho)\right], \\
\frac{d^{0.8} u_{2}}{d t^{0.8}}=-u_{2}-1.79 u_{1} u_{3}+\kappa_{2}\left[u_{2}(t)-u_{2}(t-\varrho)\right], \\
\frac{d^{0.8} u_{3}}{d t^{0.8}}=4+a_{1} u_{1} u_{2} .
\end{array}\right.
$$

Clearly, system (4.1) possesses the equilibrium point $(-1.4949,1.4949,0.5587)$. Let $\kappa_{1}=$ $\kappa_{2}=1$. Then the critical frequency $\phi_{0}=0.3944$ and the bifurcation point $\varrho_{0}=1.1844$. Then all the conditions $(\mathcal{A} 1)-(\mathcal{A} 3)$ of Theorem 3.1 hold true. Figure 2 reveals that the equilibrium point $(-1.4949,1.4949,0.5587)$ of system $(4.1)$ is locally asymptotically stable for $\varrho \in[0,1.1844)$. Figure 3 manifests that system (4.1) loses its stability and a Hopf bifurcation takes place when $\varrho \in[1.1844,+\infty)$. The relationship of the three parameters $p, \phi_{0}$ and $\varrho_{0}$ of (4.1) is clearly presented in Table 1 .

\section{Conclusions}

In this article, based on earlier studies, we propose a new fractional-order financial model. By designing a suitable time-delayed feedback controller, the chaotic behavior of the fractional-order financial model has been controlled. By adding the linear time-delayed feedback controller to both equations of fractional-order financial model and choosing the time delay as bifurcation parameter, we establish the sufficient conditions ensuring the stability and the existence of a Hopf bifurcation of a controlled fractional-order financial model. The investigation reveals that the equilibrium point of the involved system is locally asymptotically stable when the delay remains in an appropriate value, while the system will lose its stability and a Hopf bifurcation will occur when the delay exceeds the critical value. The study also shows that fractional-order and time delay have an important influence on the stability and the Hopf bifurcation of the controlled fractional-order financial model. The obtained results can help us grasp the laws of finance and interpret economical phenomena in theory. 

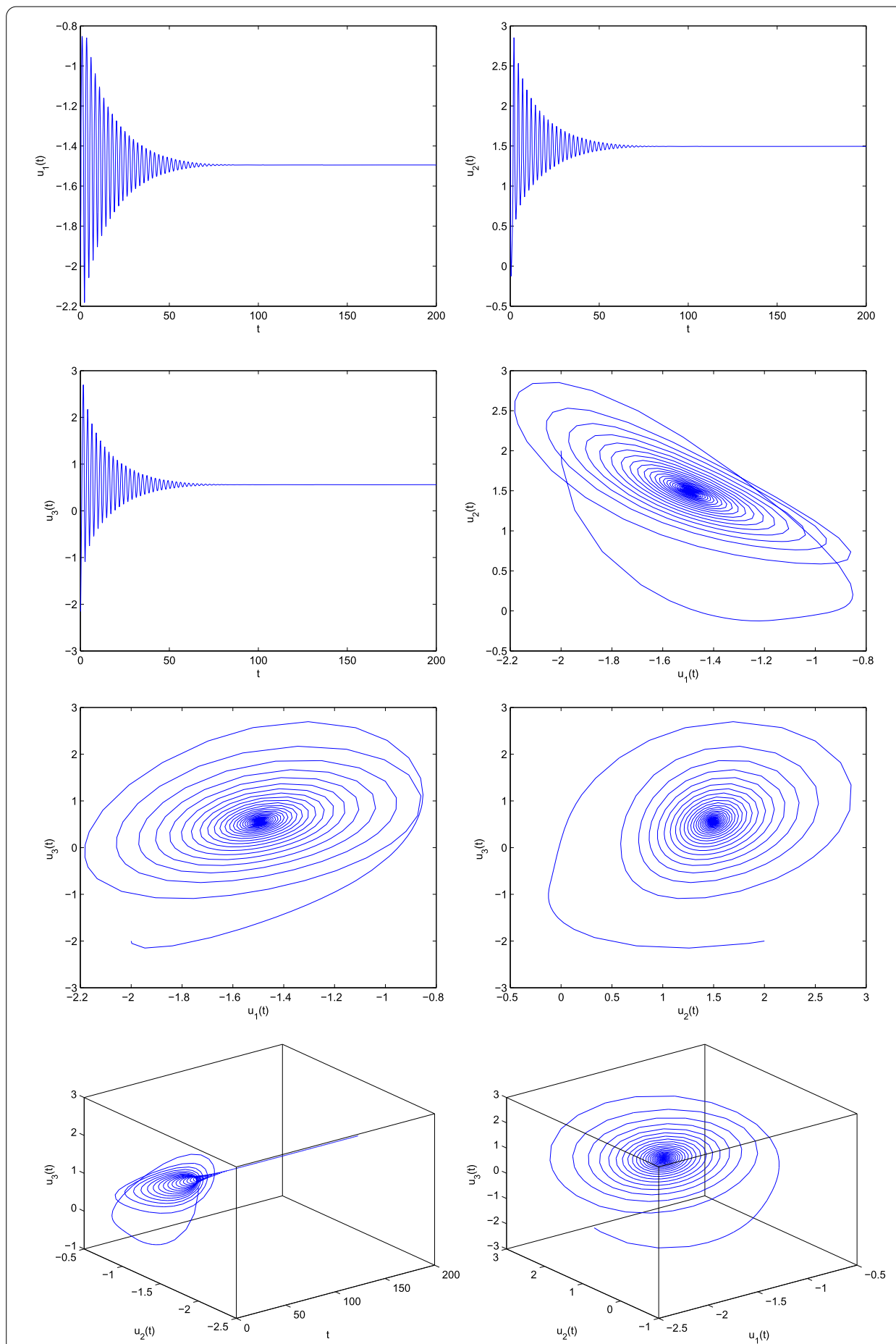

Figure $2 \varrho=1.15<\varrho_{0}=1.1844$. Time history plots, variable relation plots and phase diagrams of system (4.1). The equilibrium point $(-1.4949,1.4949,0.5587)$ of system (4.1) is asymptotically stable 

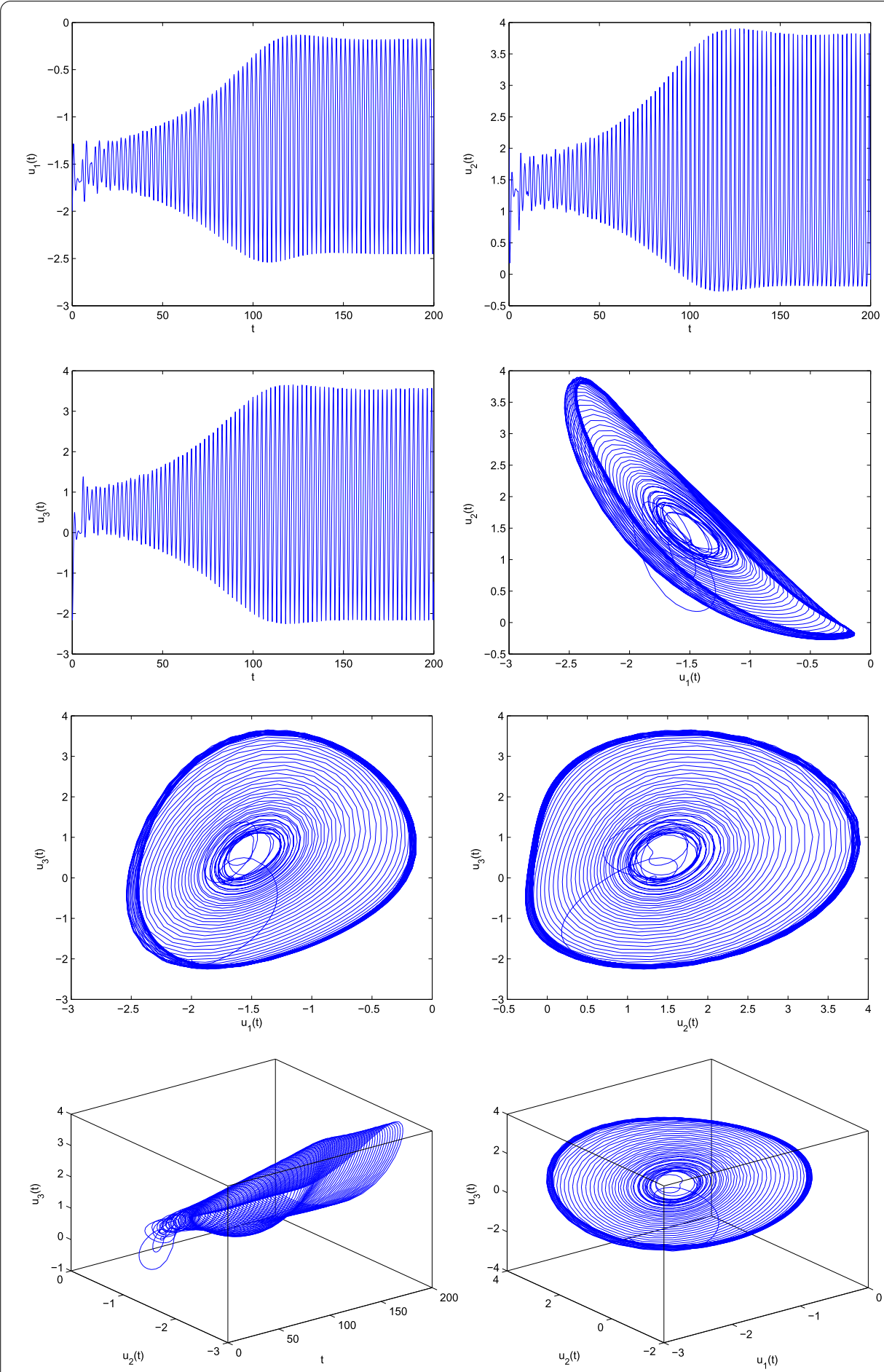

Figure $3 \varrho=1.2677>\varrho_{0}=1.1844$. Time history plots, variable relation plots and phase diagrams of system (4.1). A Hopf bifurcation of system (4.1) takes place around the equilibrium point $(-1.4949,1.4949,0.5587)$ 
Table 1 The relation of the three parameters $p, \phi_{0}, \varrho_{0}$ of (4.1)

\begin{tabular}{lll}
\hline$p$ & $\phi_{0}$ & $\varrho_{0}$ \\
\hline 0.24 & 1.8435 & 0.4377 \\
0.29 & 1.6087 & 0.5171 \\
0.31 & 1.4811 & 0.5479 \\
0.42 & 1.2062 & 0.7092 \\
0.49 & 1.0134 & 0.8054 \\
0.52 & 0.8913 & 0.8453 \\
0.67 & 0.7426 & 1.0337 \\
0.78 & 0.5866 & 1.1619 \\
0.80 & 0.3944 & 1.1844 \\
0.94 & 0.1699 & 1.3360 \\
\hline
\end{tabular}

\section{Acknowledgements}

The authors would like to thank the referees and the editor for helpful suggestions, incorporated into this paper.

\section{Funding}

The work is supported by National Natural Science Foundation of China (No.61673008 and No.62062018) and Project of High-level Innovative Talents of Guizhou Province ([2016]5651) and Major Research Project of The Innovation Group of The Education Department of Guizhou Province ([2017]039), Innovative Exploration Project of Guizhou University of Finance and Economics ([2017]5736-015), Key Project of Hunan Education Department (17A181), Hunan Provincial Key Laboratory of Mathematical Modeling and Analysis in Engineering (Changsha University of Science \& Technology) (2018MMAEZD21), University Science and Technology Top Talents Project of Guizhou Province (KY[2018]047), Guizhou University of Finance and Economics (2018XZD01) and Foundation of Science and Technology of Guizhou Province ([2019]1051).

\section{Availability of data and materials}

Data sharing not applicable to this paper as no datasets were generated or analyzed during the current study.

\section{Competing interests}

The authors declare that they have no competing interests.

\section{Authors' contributions}

All authors have read and approved the final manuscript.

\section{Author details}

'Guizhou Key Laboratory of Economics System Simulation, Guizhou University of Finance and Economics, Guiyang 550004, P.R. China. ${ }^{2}$ UR13ES47 Research Units of Mathematics and Applications, Faculté des Sciences de Bizerte, Université de Carthage, 7021, Bizerte, Tunisie. ${ }^{3}$ School of Mathematics and Physics, University of South China, Hengyang 421001 , P.R. China. ${ }^{4}$ School of Mathematics and Statistics, Henan University of Science and Technology, Luoyang 471023, P.R. China. ${ }^{5}$ School of Mathematics and Statistics, Guizhou University of Finance and Economics, Guiyang 550004, P.R. China.

\section{Publisher's Note}

Springer Nature remains neutral with regard to jurisdictional claims in published maps and institutional affiliations.

Received: 14 February 2020 Accepted: 23 September 2020 Published online: 14 October 2020

\section{References}

1. Gao, Q., Ma, J.H.: Chaos and Hopf bifurcation of a finance system. Nonlinear Dyn. 58, 209-216 (2009)

2. Ma, J.H., Chen, Y.S.: Study for bifurcation topological structure and the global complicated character of a kind of nonlinear finance system (I). Appl. Math. Mech. 22(11), 1240-1251 (2001)

3. Ma, J.H., Chen, Y.S.: Study for bifurcation topological structure and the global complicated character of a kind of nonlinear finance system (II). Appl. Math. Mech. 22(12), 1375-1382 (2001)

4. Serletic, A.: Is there chaos in economic time series? Can. J. Econ. 29, S210-S212 (1996)

5. Ott, E., Grebogi, C., Yorke, J.A.: Controlling chaos. Phys. Rev. Lett. 64, 1196-1205 (1990)

6. Pyragas, K.: Continuous control of chaos by selfcontrolling feedback. Phys. Lett. A 170, 421-429 (1992)

7. Yang, X.J., Song, Q.K., Liu, Y.R., Zhao, Z.J.: Finite-time stability analysis of fractional-order neural networks with delay. Neurocomputing 152, 19-26 (2015)

8. Deng, W.H., Li, C.P., Lü, J.H.: Stability analysis of linear fractional differential system with multiple time delays. Nonlinear Dyn. 48(4), 409-416 (2007)

9. Rakkiyappan, R., Velmurugan, G., Cao, J.D.: Stability analysis of fractional-order complex-valued neural networks with time delays. Chaos Solitons Fractals 78, 297-316 (2015)

10. Velmurugan, G., Rakkiyappan, R., Vembarasan, V., Cao, J.D., Alsaedi, A.: Dissipativity and stability analysis of fractional-order complex-valued neural networks with time delay. Neural Netw. 86, 42-53 (2017)

11. Li, M.M., Wang, J.R.: Exploring delayed Mittag-Leffler type matrix functions to study finite time stability of fractional delay differential equations. Appl. Math. Comput. 324, 254-265 (2018) 
12. Wang, Y., Jiang, J.Q.: Existence and nonexistence of positive solutions for the fractional coupled system involving generalized $p$-Laplacian. Adv. Differ. Equ. 2017, 337 (2017)

13. Zhang, J., Lou, Z.L., Jia, Y.J., Shao, W.: Ground state of Kirchhoff type fractional Schrödinger equations with critical growth. J. Math. Anal. Appl. 462(1), 57-83 (2018)

14. Wang, Y.Q., Liu, L.S.: Positive solutions for a class of fractional 3-point boundary value problems at resonance. Adv. Differ. Equ. 2017, 7 (2017)

15. Zuo, M.Y., Hao, X.A., Liu, L.S., Cui, Y.J.: Existence results for impulsive fractional integro-differential equation of mixed type with constant coefficient and antiperiodic boundary conditions. Bound. Value Probl. 2017, 161 (2017)

16. Zhang, X.G., Liu, L.S., Wu, Y.H., Wiwatanapataphee, B.: Nontrivial solutions for a fractional advection dispersion equation in anomalous diffusion. Appl. Math. Lett. 66, 1-8 (2017)

17. Feng, Q.H., Meng, F.W.: Traveling wave solutions for fractional partial differential equations arising in mathematical physics by an improved fractional Jacobi elliptic equation method. Math. Methods Appl. Sci. 40(10), 3676-3686 (2017)

18. Zhu, B., Liu, L.S., Wu, Y.H.: Existence and uniqueness of global mild solutions for a class of nonlinear fractional reaction-diffusion equations with delay. Comput. Math. Appl. 78(6), 1811-1818 (2019)

19. Yang, X.J., Song, Q.K., Liu, Y.R., Zhao, Z.J.: Finite-time stability analysis of fractional-order neural networks with delay. Neurocomputing 152, 19-26 (2015)

20. Huang, C.D., Cao, J.D.: Impact of leakage delay on bifurcation in high-order fractional BAM neural networks. Neural Netw. 98, 223-235 (2018)

21. Huang, C.D., Cao, J.D., Xiao, M.: Hybrid control on bifurcation for a delayed fractional gene regulatory network. Chaos Solitons Fractals 87, 19-29 (2016)

22. Huang, C.D., Cao, J.D., Xiao, M., Alsaedi, A., Hayat, T.: Bifurcations in a delayed fractional complex-valued neural network. Appl. Math. Comput. 292, 210-227 (2017)

23. Abdelouahab, M.S., Hamri, N.E., Wang, J.W.: Hopf bifurcation and chaos in fractional-order modified hybrid optical system. Nonlinear Dyn. 69(1-2), 275-284 (2012)

24. Rakkiyappan, R., Udhayakumar, K., Velmurugan, G., Cao, J.D., Alsaedi, A.: Stability and Hopf bifurcation analysis of fractional-order complex-valued neural networks with time delays. Adv. Differ. Equ. 2017, 225 (2017)

25. Xiao, M., Zheng, W.X., Lin, J.X., Jiang, G.P., Zhao, L.D.: Fractional-order PD control at Hopf bifurcation in delayed fractional-order small-world networks. J. Franklin Inst. 354(17), 7643-7667 (2017)

26. Xiao, M., Jiang, G.P., Zheng, W.X., Yan, S.L., Wan, Y.H., Fan, C.X.: Bifurcation control od a fractional-order van der Pol oscillator based on the state feedback. Asian J. Control 17(5), 1755-1766 (2015)

27. Ruzhansky, M., Cho, Y.J., Agarwal, P., Area, I.: Advances in Real and Complex Analysis with Applications. Springer, Singapore (2017)

28. Alderremy, A.A., Saad, K.M., Agarwal, P., Aly, S., Jain, S.: Certain new models of the multi space-fractional Gardner equation. Phys. A, Stat. Mech. Appl. 545, 123806 (2020)

29. Agarwal, P., Baleanu, D., Chen, Y., Momani, S., Machado, J.A.T.: Fractional Calculus: ICFDA 2018, Amman, Jordan, July 16-18. Springer Proceedings in Mathematics Statistics, vol. 303 (2020)

30. Agarwal, P., Agarwal, R.P., Ruzhansky, M.: Special Functions and Analysis of Differential Equations, 1st edn. CRC Press, Boca Raton (2020)

31. Agarwal, P., Hyder, A.A., Zakarya, M.: Well-posedness of stochastic modified Kawahara equation. Adv. Differ. Equ. 2020, 18 (2020)

32. Agarwal, P., Hyder, A.A., Zakarya, M., AlNemer, G., Cesarano, C., Assante, D.: Exact solutions for a class of Wick-type stochastic (3 + 1)-dimensional modified Benjamin-Bona-Mahony equations. Axioms 8(4), 134 (2019)

33. El-Sayed, A.A., Agarwal, P.: Numerical solution of multiterm variable-order fractional differential equations via shifted Legendre polynomials. Math. Methods Appl. Sci. 42(11), 3978-3991 (2019)

34. Agarwal, P., Deniz, S., Jain, S., Alderremy, A.A., Aly, S.: A new analysis of a partial differential equation arising in biology and population genetics via semi analytical techniques. Phys. A, Stat. Mech. Appl. 542, 122769 (2020)

35. Agarwal, P., Singh, R.: Modelling of transmission dynamics of Nipah virus (Niv): a fractional order approach. Phys. A, Stat. Mech. Appl. 547, 124243 (2020)

36. Tang, X.H., Chen, S.T., Lin, X.Y., Yu, J.S.: Ground state solutions of Nehari-Pankov type for Schrödinger equations with local super-quadratic conditions. J. Differ. Equ. 268(8), 4663-4690 (2020)

37. Tang, X.H., Chen, S.T.: Singularly perturbed Choquard equations with nonlinearity satisfying Berestycki-Lions assumptions. Adv. Nonlinear Anal. 9(1), 413-437 (2020)

38. Huang, C.X., Zhang, H., Huang, L.H.: Almost periodicity analysis for a delayed Nicholson's blowflies model with nonlinear density-dependent mortality term. Commun. Pure Appl. Anal. 18(6), 3337-3349 (2019)

39. Huang, C.X., Yang, Z.C., Yi, T.S., Zou, X.F.: On the basins of attraction for a class of delay differential equations with non-monotone bistable nonlinearities. J. Differ. Equ. 256(7), 2101-2114 (2014)

40. Ahamad, H., Mojtaba, H., Dumitru, B.: On the adaptive sliding mode controller for a hyperchaotic fractional-order financial system. Phys. A, Stat. Mech. Appl. 497, 139-153 (2018)

41. Jajarmi, A., Hajipour, M., Baleanu, D.: New aspects of the adaptive synchronization and hyperchaos suppression of a financial model. Chaos Solitons Fractals 48(33), 285-296 (2017)

42. Duan, L., Fang, X.W., Huang, C.X.: Global exponential convergence in a delayed almost periodic Nicholson's blowflies model with discontinuous harvesting. Math. Methods Appl. Sci. 41(5), 1954-1965 (2018)

43. Huang, C.X., Yang, X.G., Cao, J.D.: Asymptotically stable high-order neutral cellular neural networks with proportional delays and D operators. Math. Comput. Simul. 171, 127-135 (2020)

44. Huang, C.X., Wen, S.G., Huang, L.H.: Dynamics of anti-periodic solutions on shunting inhibitory cellular neural networks with multi-proportional delays. Neurocomputing 357, 47-52 (2019)

45. Wang, W.T., Liu, F.Y., Chen, W.: Exponential stability of pseudo almost periodic delayed Nicholson-type system with patch structure. Math. Methods Appl. Sci. 42(2), 592-604 (2019)

46. Wang, W.T.: Finite-time synchronization for a class of fuzzy cellular neural networks with time-varying coefficients and proportional delays. Fuzzy Sets Syst. 338, 40-49 (2018)

47. Wang, W.T., Chen, W.: Stochastic Nicholson-type delay system with regime switching. Syst. Control Lett. 136, 104603 (2020) 
48. Wang, W.T., Wang, L.Q., Chen, W.: Stochastic Nicholson's blowflies delayed differential equations. Appl. Math. Lett. 87, 20-26 (2019)

49. Yang, J.H., Zhang, E.L., Liu, M.: Bifurcation analysis and chaos control in modified finance system with delayed feedback. Int. J. Bifurc. Chaos 26(6), 1650105 (2016)

50. Podlubny, I.: Fractional Differential Equations. Academic Press, New York (1999)

51. Matignon, D.: Stability results for fractional differential equations with applications to control processing. In: Computational Engineering in Systems and Application Multi-Conference, IMACS, Lille, France, July 1996. IEEE-SMC Proceedings, vol. 2, pp. 963-968 (1996)

52. Yu, P., Chen, G.R.: Hopf bifurcation control using nonlinear feedback with polynomial functions. Int. J. Bifurc. Chaos 14(5), 1683-1704 (2004)

53. $\mathrm{Ma}, \mathrm{C}$. ., Wang, X.Y.: Hopf bifurcation and topological horseshoe of a novel finance chaotic system. Commun. Nonlinear Sci. Numer. Simul. 17, 721-730 (2012)

54. Xu, C.J., Zhang, Q.M.: On the chaos control of the Qi system. J. Eng. Math. 90(1), 67-81 (2015)

\section{Submit your manuscript to a SpringerOpen ${ }^{\mathcal{O}}$} journal and benefit from:

- Convenient online submission

- Rigorous peer review

- Open access: articles freely available online

- High visibility within the field

- Retaining the copyright to your article

Submit your next manuscript at $\gg$ springeropen.com 